Finite-dimensional behaviour and observability in a randomly forced PDE

Broomhead, David and Huke, Jeremy and Montaldi, James and Muldoon, Mark

2012

MIMS EPrint: 2012.69

Manchester Institute for Mathematical Sciences

School of Mathematics

The University of Manchester

\footnotetext{
Reports available from: http://eprints.maths.manchester.ac.uk/

And by contacting: The MIMS Secretary

School of Mathematics

The University of Manchester

Manchester, M13 9PL, UK
} 


\title{
FINITE-DIMENSIONAL BEHAVIOUR AND OBSERVABILITY IN A RANDOMLY FORCED PDE
}

\author{
D.S. BROOMHEAD, J.P. HUKE, J. MONTALDI, M.R. MULDOON
}

\begin{abstract}
In earlier work [5], aimed at developing an approach to signal processing that can be applied as well to nonlinear systems as linear ones, we produced mathematical models of digital communications channels that took the form of iterated function systems (IFS). For finite-dimensional systems these models have observability properties indicating they could be used for signal processing applications.

Here we see how far the same approach can be taken towards the modelling of an infinite-dimensional system. The cable equation is a well-known partial differential equation model of an imperfectly insulated uniform conductor, coupled to its surroundings by capacitive effects. (It is also much used as a basic model in theoretical neurobiology.) In this paper we study the dynamics of this system when it is subjected to randomly selected discrete input pulses. The resulting IFS has a unique finite-dimensional attractor; we use results of Falconer and Solomyak to investigate the dimension of this attractor, relating it to the physical parameters of the system. Using work of Robinson, we show how some of the observability properties of the IFS model are retained.
\end{abstract}

\section{INTRODUCTION}

Traditional theoretical approaches to signal processing rely very heavily on linear systems theory. However, some systems arising in applications involve significant nonlinearities: these occur in, for example, satellite digital transmission and fibreoptic systems. We would therefore like to develop an approach that treats linear and nonlinear systems on an equal footing. In [5] we describe such an approach relevant to the particular area of digital communications; 'digital' here implies that the message to be communicated consists of a sequence of symbols, all taken from a fixed, finite set (or 'alphabet'). The ideas described in [5] are motivated by a fundamentally physical view of signal transmission: a signal is sent by creating an excitation at some location (the transmitter) in a physical medium, and allowing it to propagate to some destination (the receiver). (For example, the medium in wireless radio transmission is the electromagnetic field in the atmosphere or free space; in sonar the medium is the ocean.) The excitation is subject to various kinds of transformation, linear or nonlinear, as it propagates (dispersion, distortion, attenuation etc.). Receiving the signal consists of detecting the excitation as it arrives - which essentially involves making local measurements on the medium - and subsequently trying to deduce the original signal that was transmitted. The medium through which the signal passes is sometimes referred to as the 'channel' (or more specifically the propagation channel). In fact, the signal may pass through several different media, and be converted to various different physical forms before it reaches the receiver; the channel can be extended to cover all these, and indeed to cover certain signal processing operations at the receiver and transmitter; the important thing from the signal processing viewpoint 
is the overall transformation that the signal undergoes between transmission and reception.

Motivated by the idea of the channel as an excited physical medium, ref. [5] treats the channel as a dynamical system. The external forcing means the dynamics are non-autonomous, but the fact that the signal is digital means that the input to the channel consists of sequences of elementary inputs, one for each symbol. To be more specific, suppose there are $N_{S}$ symbols in the alphabet (for the common case of binary signals, $N_{S}=2$ ). We assume that while the signal is being sent time is partitioned into intervals of fixed length $\tau$ (the 'symbol period'), each interval corresponding to the transmission of a single symbol. During a symbol period the system evolves according to a certain vector field; this is generally time-varying, because of the forcing by the transmitter. The vector field obviously depends on the forcing, and hence on the particular symbol being transmitted, but the finiteness of the alphabet means there is correspondingly only a finite number of possibilities for the field. Thus if the system is in state $\boldsymbol{x}$ at the beginning of the symbol period, there are only finitely many states it can be in at the end, and these are found by integrating the various vector fields (one for each symbol) with $\boldsymbol{x}$ as initial condition.

The mathematical structure which this picture suggests is that of an iterated function system (IFS): a discrete time, random dynamical system, consisting of a finite family of mappings of the state space to itself, one of which is chosen at random at each time step. (The system is discrete time because we focus on how the state has changed over a symbol period, ignoring the details of what happens within a period.)

The receiver cannot generally identify the state of the system (i.e. the channel) completely, but instead can make measurements on it, (which typically consist of measuring in some way the level of excitation local to receiver). The measurement procedure is represented by a real-valued function on the state space: each state of the medium gives rise to a corresponding measurement value. We assume for definiteness that the receiver determines the value of this function at the end of each symbol period. (The measurement is determined completely by the state of the system when the measurement is made, but note that, because of effects such as dispersion, the state - and the measurement-will typically be influenced by all the symbols that have been transmitted up to the time the measurement is made.) It is important to know whether the states of the system are fixed uniquely by finite sequences of these values (a situation known as 'observability'); in [5, 16] we give observability results for the case where the state space is finite-dimensional. It is assumed that the dynamics of the channel (and so the maps in the IFS) are differentiable, but it is not assumed that they are linear.

Traditional signal processing commonly assumes that the channel is a finite-dimensional system, but often the most natural description of the passage of a signal through a medium involves partial differential equations - and correspondingly, an infinitedimensional system: electromagnetic propagation through free space or other media, and sound propagation in the atmosphere or ocean are obvious examples. Although such systems are infinite-dimensional, this does not really change the dynamical systems viewpoint outlined above: in particular, the IFS structure is retained. Can we carry the ideas in [5] over to such infinite-dimensional systems, and so broaden the approach to include this range of digital communications scenarios? In this paper we describe how to do this, largely by considering a simple example: the 'cable equation' described below. 
The main difficulty in applying the ideas of [5] to infinite-dimensional systems is that the observability results relating sequences of measurements to the underlying states rely on the finite dimensionality of the state space. We can try to get around this problem by considering only states in the attractor of the IFS, rather than all possible states. As discussed in [5], fairly broad physical considerations lead us to expect that the IFS should have an attractor; for finite-dimensional systems this attractor must clearly itself have finite dimension, but it is possible the attractor has finite dimension even if the system itself is infinite-dimensional. If so, then the attractor may possess the kind of observability property that the receiver needs to gain information about the transmitted symbol sequence.

\section{THE CABLE EQUATION}

In this paper we study a specific partial differential equation, and an IFS to which it gives rise, and show that the attractor for the IFS indeed has finite dimension; we find the dimension in terms of the system's parameters.

The equation that we shall study is the cable equation: a parabolic PDE that models the space and time behaviour of the voltage in an essentially one dimensional conductor, such as a length of wire. The equation was first proposed by Kelvin [19] as a model to describe signals transmitted along undersea telegraph cables. Its applicability to general transmission line problems is limited by the fact that it takes no account of inductive effects - but we nevertheless adopt it for illustrative purposes. In fact, the cable equation now enjoys some prominence because there have been a range of neurobiological models where it is used to model propagation along nerve cylinders such as dendrites or unmyelinated axons [20,6].

We can think of the cable as consisting of a conducting core, surrounded by a (partially) insulating layer - which for a nerve cylinder is the cell membrane -itself surrounded by another conductor or conducting medium. (The cable equation also applies to other geometries [11].) The external medium is assumed to be at a uniform voltage which can be taken to be zero. The core conductor has a uniform resistance, $R$, per unit length; the insulating layer provides a capacitance of $C$ per unit length. The insulator is further assumed to have a uniform leakage conductance, $A$, per unit length. (In the neurobiological context, it is assumed that the local voltage is small enough for this conductance to be voltage-independent - the membrane is 'subthreshold'. At larger voltages the conductance varies with voltage, leading to nonlinear effects such as action potentials; the cable equation is not suitable for describing these.)

We shall take the cable to have a finite length $l$, with ends at $x=0$ and $x=l$. The basic physical assumptions just described can be used to derive the cable equation (see [20] for details):

$$
C \frac{\partial v}{\partial t}=\frac{1}{R} \frac{\partial^{2} v}{\partial x^{2}}-A v+I_{e}
$$

where $v(x, t)$, gives the voltage at time $t$ as a function of distance $x$ along the cable. $I_{e}(x, t)$ is an input current density (i.e. input current per unit length of cable): in neurobiological applications this current density may arise from an externally applied current (via one or more electrodes), or through synaptic input $[20,6]$. It is through this term that our signal is introduced into the channel. 
Returning for a moment to the transmission line scenario, it is clear that signal input will be localized close to one end (the 'transmitter end') of the cable ( In fact the most obvious way to introduce the signal would be to constrain the voltage at the transmitter end to follow some prescribed time variation - that is, the signal is input by choosing a time varying boundary condition at $x=0$ (this is the approach described by Kelvin [19] - the receiver end is taken to be grounded). $I_{e}$ would then be absent from (1), and the equation would be homogeneous. However, it will turn out to be convenient for us to use fixed boundary conditions whatever signal we wish to send. We can arrange this by the standard device of expressing the solution as the sum of a function satisfying the boundary conditions and a function satisfying a suitably modified PDE - that is, swapping a homogeneous equation with inhomogeneous boundary conditions for an inhomogeneous equation with homogeneous boundary conditions. In this scenario, $I_{e}$ is to be interpreted as the corresponding inhomogeneous term rather than a physical input current.

For illustrative purposes we will take the boundary conditions to be Dirichlet: $v(0, t)=v(l, t)=0$. (Tuckwell [20] describes various boundary conditions, and their interpretations in the nerve cylinder model; the Dirichlet boundary conditions used here represent 'killed end' termination of the nerve cylinder.)

If we introduce new space and time variables $x \mapsto x / l, t \mapsto t /\left(l^{2} R C\right)$ and a new parameter $\rho=l^{2} R A$, the cable equation becomes

$$
\partial_{t} v=\partial_{x}^{2} v-\rho v+J_{e}
$$

where $J_{e}(x, t)=l^{2} R I_{e}(x, t)$. (We will continue to denote the symbol period, in the new time units, by $\tau$.)

As mentioned above, the current density $I_{e}(x, t)$ provides the mechanism by which we input the symbol sequence to the channel. Following the discussion in the Introduction, we partition time into intervals $[n \tau,(n+1) \tau)$, and within each such interval $I_{e}:[0, l] \times[n \tau,(n+1) \tau) \rightarrow \mathbb{R}$ takes one of a finite number of prescribed forms. We label these forms $I_{e k}$ (with corresponding $J_{e k}$ ), $k=1,2, \ldots, N_{S}$ where $N_{S}$ is the number of different symbols. Each of these produces one mapping in the corresponding IFS, as described in the next section.

\section{ConstruCting THE ITERATED FUnCTION SYSTEM}

As described in the Introduction (and in more detail in [5]) the state of the channel is represented by a point $\boldsymbol{z}$ in a state space $M$; in [5] $M$ is a finite-dimensional manifold, and if the $k$-th symbol is transmitted during the symbol period $[0, \tau)$ then $\boldsymbol{z}$ evolves according to

$$
\dot{\boldsymbol{z}}=F_{k}(\boldsymbol{z}, t)
$$

where $F_{k}: M \times[0, \tau) \rightarrow T M$ is the time-dependent vector field corresponding to the $k$-th symbol. The $k$-th map $f_{k}: M \rightarrow M$ is constructed by setting $f_{k}\left(\boldsymbol{z}_{0}\right)$ equal to $\boldsymbol{z}(\tau)$, found by integrating (3) over the range $[0, \tau]$ with $\boldsymbol{z}_{0}$ as initial condition.

We need a mathematical framework that allows us to apply these concepts to infinite-dimensional systems; one such framework is the theory of strongly continuous semigroups (or $C_{0}$ semigroups). We will not attempt to cover this theory hereaccessible introductions can be found in $[4,13]$ - contenting ourselves with merely sketching how it applies to our current example. 
The first part of the picture that we need to extend to the infinite dimensional setting is the state space. For systems described by PDEs, the state of the system at any given time is specified by one or more functions - in the cable example, the state at time $t$ is the voltage profile along the cable $v(x, t)$ ( $t$ regarded as fixed). Thus the state space will be a function space $(X$, say), and the trajectory will be a mapping from the time axis into $X, u:[0, \tau] \rightarrow X$, with $u(t)=v(\cdot, t) . u(t)$ corresponds to $\boldsymbol{z}(t)$ in the above paragraph, and there will be a corresponding evolution equation (a PDE) playing the role of equation (3) - for the cable example this equation is (2). (Ultimately the mappings of the IFS will be found as before: for the $k$-th symbol there is a map $f_{k}: X \rightarrow X$, found by solving the PDE (which will depend on $k$ ) with a given initial condition, and evaluating the solution at time $\tau$.)

In general, then, we shall take the state space to be a Banach space, $X$. The theory of $C_{0}$ semigroups can be applied to (among other things) differential equations in Banach spaces; various kinds of differential equation can be treated using this theory (including certain nonlinear kinds) but here we are interested in the linear, nonhomogeneous abstract Cauchy problem:

$$
\begin{array}{rlr}
\dot{u} & =A u+g \quad t>0 \\
u(0) & =w
\end{array}
$$

where $u$ and $g$ are mappings from the time axis into $X, A$ is a linear operator whose domain $D(A)$ is a linear subspace of $X, A: D(A) \subset X \rightarrow X$, and the initial state $w \in X$. The cable equation can be put in this form by interpreting $u(t)=v(\cdot, t)$ as above, (and similarly $g(t)=J_{e k}(\cdot, t)$ ), and taking $A$ to be the differential operator $\partial_{x}^{2}-\rho I$.

Consider first the autonomous version of (4), with $g \equiv 0$. If $A$ satisfies certain conditions $[4,13]$, there is a collection of (continuous) linear operators $S(t): X \rightarrow X$, one for each time $t$, having the properties $S(0)=I$ (the identity), and $S(t+s)=$ $S(t) S(s)$ for all $t, s \geq 0$. ( $A$ is the so-called infinitesimal generator of the semigroup $\{S(t)\}_{t \geq 0}$.) Furthermore, if the initial condition $w \in D(A)$, the function $u(t)=S(t) w$ satisfies the homogeneous problem $\dot{u}=A u$ for all $t \geq 0$ (and in particular, $u(t) \in$ $D(A)$ for all $t$ ). (In the finite-dimensional case, these operators would be referred to as the 'time $t$ maps' of the autonomous system.)

Returning to the inhomogeneous equation (4), it can be shown [13] that any solution having initial condition $w \in H$ must have the standard 'variation of parameters' form:

$$
u(t)=S(t) w+\int_{0}^{t} S(t-s) g(s) d s
$$

(where it has been assumed that $g(t)$ is sufficiently well behaved that the integral on the right hand side exists). This form can encompass more than just the ordinary solutions: the function $u(t)$ specified by (5) can exist without $u$ being a smooth enough function of time to qualify as a solution of (4); there is then no solution in the strict sense but we may nevertheless choose to regard $u$ as a 'generalized solution' - it is referred to as the mild solution. Conditions on $t \rightarrow g(t)$ (essentially smoothness conditions) that ensure that the solutions given by (5) are in fact strict solutions of (4) are given in $[4,13]$.

Let us apply these general considerations to the cable example. Note to begin with that for infinite-dimensional systems the choice of state space is a more subtle problem than is usually the case for finite-dimensional systems: the function space may 
depend on, for example, the boundary conditions, the regularity (e.g. smoothness) of non-homogeneous terms, and the regularity that we require our solutions to have. Choosing this state space is part of the modelling problem for the physical system we are dealing with: these matters are discussed in [4] and [21]. Our digital signalling application imposes one or two further requirements: we shall need the state space to be the same for all of the symbols, even though the PDE varies with the symbol (this is why we arranged above for all the PDEs to have the same boundary conditions); and we also need the maps in the IFS to be contractions.

For the cable example, one possible choice for the state space $X$ is the space $L^{2}(0,1)$, consisting of square integrable functions on $(0,1)$. $A$ is the differential operator $\partial_{x}^{2}-\rho I$ : the domain of this operator is not the whole state space $H$-indeed we shall take the domain to be

$$
D(A)=\left\{w \in L^{2}(0,1): w^{\prime} \in L^{2}(0,1), w^{\prime \prime} \in L^{2}(0,1), w(0)=w(1)=0\right\}
$$

(The derivatives here are taken to be weak derivatives. Note that the Dirichlet boundary conditions are incorporated into the definition of $D(A)$, and are thus taken into account because we seek solutions such that $u(t) \in D(A)$ for $t>0$.) It can be shown [21] that with these choices of $A$ and $D(A), A$ is the infinitesimal generator for a $C_{0}$ semigroup $\{S(t)\}_{t \geq 0}$ on $L^{2}(0,1)$; and further, that this semigroup has the property $\|S(t) w\| \leq e^{-\left(\rho+\pi^{2}\right) t}\|w\|$ for all $w \in L^{2}(0,1)$, where $\|\cdot\|$ means the $L^{2}$ norm.

Expression (5) now furnishes the required solutions of the PDE, as trajectories in the state space $X$. We may thus construct the maps in the IFS as we did in the finitedimensional case. During each symbol period the system is governed by an equation in the form of (4) - we should now provide $g$ with a subscript $k$ indicating which symbol is being transmitted. (In the cable equation $A$ turns out to be independent of $k$, but the procedure does not require this, and $A$ could vary with $k$ in other systems.) The $k$-th map of the IFS is given by $f_{k}(w)=u(\tau)$, where $u(\tau)$ is found from $(5)$.

To see that $f_{k}$ is a contraction, note that only the first term of (5) depends on the initial state, so given two initial states $w_{1}$ and $w_{2}$, the corresponding solutions satisfy $u_{2}(t)-u_{1}(t)=S(t)\left(w_{2}-w_{1}\right)$, and hence $\left\|u_{2}(t)-u_{1}(t)\right\| \leq e^{-\left(\rho+\pi^{2}\right) t}\left\|w_{2}-w_{1}\right\|$. Setting $t=\tau$ we see that $f_{k}$ is a contraction mapping, with contraction factor $e^{-\left(\rho+\pi^{2}\right) \tau}$.

\section{EXISTENCE AND UNIQUENESS OF THE ATTRACTOR}

For an infinite-dimensional system we generally expect the state space to be a Banach space, $X$, and thus a complete metric space. For such a space we denote the collection of non-empty bounded sets by $\mathcal{B}$; we can supply $\mathcal{B}$ with a notion of distance by using the Hausdorff distance [10] $\mathbf{d}_{H}: \mathcal{B} \times \mathcal{B} \rightarrow \mathbb{R}^{+}$. $\mathbf{d}_{H}$ is not a metric on $\mathcal{B}$ because a set $A \in \mathcal{B}$ may be different from its closure $\bar{A}$, while nevertheless $\mathbf{d}_{H}(A, \bar{A})=0$. In fact, for two sets $A$ and $B$ in $\mathcal{B}, \mathbf{d}_{H}(A, B)=0$ if and only if $\bar{A}=\bar{B}$. $\mathbf{d}_{H}$ does, however, satisfy the triangle inequality. If we consider the collection $\mathcal{C}$ of non-empty compact subsets of $X$, then $\mathcal{C}$ does turn out to be a complete metric space when supplied with the metric $\mathbf{d}_{H}[2,7]$.

The finite union of contractions of any bounded set is also bounded; so, given a collection of contractions $f_{k}: X \rightarrow X, k=1, \ldots, N_{S}$, the map $F: \mathcal{B} \rightarrow \mathcal{B}$ specified 
by

$$
F U=\bigcup_{k=1}^{N_{S}} f_{k} U
$$

is well-defined. For any $A \in \mathcal{C}$ we have $F A \in \mathcal{C}$, and a well-known $[2,7,10]$ theorem asserts that $F$, when restricted to $\mathcal{C}$, has a unique fixed point, $K$; that is, $K$ is a unique, non-empty compact subset of $X$ which satisfies

$$
K=\bigcup_{k=1}^{N_{S}} f_{k} K
$$

Moreover, for any $A \in \mathcal{C}$, the sequence $F^{n} A$ converges, in the Hausdorff metric, to $K ; K$ may therefore be referred to as the attractor for the map $F$.

We saw in the previous section that for the cable example, the maps $f_{k}$ are all contractions with contractivity factor $s=e^{-\left(\rho+\pi^{2}\right) \tau}$; hence the discretely-forced cable equation system has a unique compact attractor, $K \subset L^{2}(0,1)$, to which all nonempty, compact subsets of $L^{2}(0,1)$ converge.

In fact, the idea that $F^{n} A$ converges to $K$ (in the Hausdorff distance sense) applies to the whole of $\mathcal{B}$ :

Proposition 1. For any $A \in \mathcal{B}$, the sequence $F^{n} A$ converges to $K$; that is, $\mathbf{d}_{H}\left(F^{n} A, K\right) \rightarrow 0$ as $n \rightarrow \infty$.

To prove this proposition, let $\mathbf{d}$ be the metric on $X$, and say that an $r$-net of a set $A \in \mathcal{B}$ is a finite set of points $\left\{y_{1}, \ldots, y_{n}\right\} \subset A$ such that for every $y \in A, \mathbf{d}\left(y, y_{i}\right)<r$ for at least one $i, 1 \leq i \leq n$. Also let $s<1$ be the largest of the contraction factors for the maps $f_{k}, k=1, \ldots, N_{S}$. Then we have the following lemma:

Lemma 2. If $A_{r}$ is an $r$-net of $A \in \mathcal{B}$ then $F A_{r}$ is an sr-net of $F A$.

Proof. Take any $z \in F A$, then there exists at least one $w \in A$ and a corresponding index $k$ such that $f_{k}(w)=z$. Since $A_{r}$ is an $r$-net of $A$ there is a $y \in A_{r}$ such that $\mathbf{d}(w, y)<r$. Then

$$
\mathbf{d}\left(z, f_{k}(y)\right)=\mathbf{d}\left(f_{k}(w), f_{k}(y)\right) \leq s \mathbf{d}(w, y)<s r
$$

and hence any $z \in F A$ lies within $s r$ of a point in $F A_{r}$.

Proof of Proposition 1. To prove Proposition 1, we need to show that for any $A \in \mathcal{B}$, $\mathbf{d}_{H}\left(F^{n} A, K\right) \rightarrow 0$ as $n \rightarrow \infty$. Since $A$ is bounded there is a point $z \in A$ and a closed ball $B_{r}(z)$ with radius $r$, such that $A \subset B_{r}(z)$. Thus $A_{r}=\{z\}$ is an $r$-net of $A$. It follows from the Lemma that $F^{n} A_{r}$ is an $s^{n} r$-net of $F^{n} A$ and so, using the triangle inequality, we have:

$$
\mathbf{d}_{H}\left(F^{n} A, K\right) \leq \mathbf{d}_{H}\left(F^{n} A, F^{n} A_{r}\right)+\mathbf{d}_{H}\left(F^{n} A_{r}, K\right)
$$

where $\mathbf{d}_{H}\left(F^{n} A, F^{n} A_{r}\right) \leq s^{n} r$ and so approaches 0 as $n \rightarrow \infty$. Note that $A_{r}$ is a finite set and so is compact; recalling that $F^{n} C \rightarrow K$ for any compact set $C$, we see that the second term on the right hand side of the inequality also tends to 0 .

Recall from the Introduction that - in an effort to transfer the ideas of [5] to the infinite-dimensional case - we hope to establish that the attractor of the IFS has finite dimension. We can now do this for IFS's consisting of contraction mappings. 
Proposition 3. The (upper) box-counting dimension, and hence the Hausdorff dimension, of $K$ is bounded:

$$
\operatorname{dim}_{H}(K) \leq \overline{\operatorname{dim}}_{B}(K) \leq \log N_{S} /|\log s| .
$$

Proof. The (upper) box-counting dimension is always an upper bound of the Hausdorff dimension, so we need only show that the former is bounded. Since $K$ is compact it has an $r$-net for every $r>0$. Let $K_{r}$ be an $r$-net of $K$, and say it contains $\bar{n}(r)$ points. Then

$$
K \subseteq \bigcup_{z \in K_{r}} B_{r}(z)
$$

and so, letting $n(r)$ be the minimum number of $r$-balls required to cover $K$, we have $\bar{n}(r) \geq n(r)$. Using Lemma 2 we can transfer this bound to smaller radii, because $F K_{r}$ is an $s r$-net of $K$; since $F K_{r}$ has (at most) $N_{S} \bar{n}(r)$ elements we have $N_{S} \bar{n}(r) \geq \bar{n}(s r) \geq n(s r)$. Proceeding recursively we get for any natural number $l$

$$
N_{S}^{l} \bar{n}(r) \geq n\left(s^{l} r\right)
$$

The upper box-counting dimension can be computed from (see e.g. [7])

$$
\operatorname{dim}_{B}(K)=\varlimsup_{l \rightarrow \infty} \frac{\log n\left(s^{l} r\right)}{\left|\log s^{l} r\right|}
$$

and so we have the required inequality:

$$
\operatorname{dim}_{B}(K) \leq \varlimsup_{l \rightarrow \infty} \frac{\log N_{S}^{l} \bar{n}(r)}{\left|\log s^{l} r\right|}=\frac{\log N_{S}}{|\log s|}
$$

\section{AN EXPLICIT FORM FOR THE ITERATED FUNCTION SYSTEM}

In both the theoretical and numerical solution of equations such as (4) an important role is played by the eigenfunctions of the operator $A$. For the cable equation, where $A=\partial_{x}^{2}-I$ (with Dirichlet boundary conditions), these eigenfunctions are simply $\sin n \pi x, n=1,2, \ldots$. The maps in the IFS take particularly simple forms if we expand the solutions in terms of these eigenfunctions (i.e. as a Fourier (sine) series):

$$
v(x, t)=\sum_{n=1}^{\infty} v_{n}(t) \sin n \pi x .
$$

We expand the input current density for the $k$-th symbol, $J_{e k}$, in the same way

$$
J_{e k}(x, t)=\sum_{n=1}^{\infty} J_{n}^{(k)}(t) \sin n \pi x .
$$

Since, for each $t$, the functions $v(\cdot, t)$ and $J_{e k}(\cdot, t)$ lie in our state space $L^{2}(0,1)$, the vectors of Fourier coefficients $\boldsymbol{v}(t)=\left(v_{1}(t), v_{2}(t), \ldots\right)$, and $\boldsymbol{J}^{(k)}(t)$ lie in $l_{2}$ (the space of square-summable sequences). We shall re-express the IFS as a collection of maps on this space; the transformation between the two state spaces is a similarity (and even an isometry if we use normalized eigenfunctions), so the geometry of the attractor (and indeed of the trajectories) is preserved. (We shall continue to refer to the attractor as $K$.) 
Substituting equations (8) and (9) into equation (2) gives the following countable system of independent ordinary differential equations:

$$
\dot{v}_{n}(t)=-\left(\rho+n^{2} \pi^{2}\right) v_{n}(t)+J_{n}^{(k)}(t), \quad k \in \mathbb{N}
$$

so that each component of $\boldsymbol{v}$ evolves independently of all the others.

If we solve these equations over one symbol period, taking each of the possible $N_{S}$ symbols as input, we obtain:

$$
\left\{v_{n} \mapsto \lambda_{n} v_{n}+b_{n}^{(k)}: n \in \mathbb{N}\right\} \quad k=1, \ldots, N_{S}
$$

where

$$
\lambda_{n}=\exp \left[-\tau\left(\rho+n^{2} \pi^{2}\right)\right] \quad \text { and } \quad b_{n}^{(k)}=e^{-\tau\left(\rho+n^{2} \pi^{2}\right)} \int_{0}^{\tau} e^{t\left(\rho+n^{2} \pi^{2}\right)} J_{n}^{(k)}(t) d t .
$$

These expressions specify (componentwise) a set of $N_{S}$ affine maps $\bar{f}_{k}: l_{2} \rightarrow l_{2}$, which we write as $\bar{f}_{k}(\boldsymbol{v})=\bar{T} \boldsymbol{v}+\boldsymbol{b}^{(k)}$. Note that the linear parts $\bar{T}$ of these maps are identical (independent of symbol); they are diagonal contractions with contraction rates given by $(12)$.

One consequence of expressing the IFS in this form is that we can draw some conclusions regarding the connectedness and 'addressability' of the attractor. Let $\Omega$ be the set of (infinite) sequences of symbols, $\left\{1, \ldots, N_{S}\right\}^{\mathbb{N}}$; for any IFS consisting of contractions, there is a mapping $\pi: \Omega \rightarrow K$ given by

$$
\left(i_{1}, i_{2}, \ldots,\right) \mapsto \lim _{n \rightarrow \infty} f_{i_{1}} \circ f_{i_{2}} \circ \ldots f_{i_{n}}(u)
$$

(where $u$ is any point on the attractor - the limit is independent of $u$ ). Hutchinson [10] showed that $\pi$ is onto and continuous ( $\Omega$ has the cylinder set topology); it need not be injective, but if it is, then it is in fact a homeomorphism, and $K$ is a totally disconnected set. The sequences assigned by $\pi$ to a given point on the attractor are the 'addresses' of that point [2] - each specifies a history of maps that bring the state of the system to the given point: early members of the address correspond to recently applied maps, later members indicate maps applied in the more distant past. The address is of particular interest in the signalling context because it specifies the history of transmitted symbols; if the addresses are unique, it is possible in principle to infer the transmitted symbol sequence, from the current position on the attractor (although limited precision will in practice restrict this to more-or-less recent symbols). If the addresses are not unique we must look at a sequence of states to infer the symbol sequence, not just one.

Returning to the cable equation system, note that for each $n,(11)$ defines an IFS on the real line, with contraction mappings having contractivity factor $\lambda_{n}$. By choosing $n$ sufficiently large we can make this factor arbitrarily small, and so (by Proposition 3 ) make the dimension of the attractor of the 1-dimensional IFS arbitrarily small-in particular, we can make it less than 1. It follows [7, Proposition 2.5] that the 1-d attractor is totally disconnected.

Total disconnectedness of an attractor does not imply uniqueness of addresses, except in the case where the IFS consists of just two maps [3], but this case is of some interest since it arises when the symbol alphabet consists (as it commonly does) of the binary digit set $\{0,1\}$. Note that unique addressability (i.e. injectivity of $\pi$ ) for the 1-d attractor implies unique addressability for $K$ itself (points on $K$ corresponding 
to different addresses must differ at least in their $n$-th coordinate). Thus in the two map case, $K$ is always totally disconnected, and addresses are unique.

If there are more than two maps the situation is more subtle. It remains true, of course, that for all sufficiently large $n$ the attractor of the $n$-th 1-dimensional IFS will be totally disconnected, but it may not be uniquely addressable. If any of the 1 -d attractors is uniquely addressable, then so is $K$-so we might suspect that this is a common situation; it is however possible to construct examples where $K$ is not uniquely addressable (this is straightforward if, for example, the expansions (9) have only finitely many terms).

\section{The DIMENSION OF $K$}

We shall now use the form of the IFS described in the previous section to deduce more precise information about the dimension of the attractor. We shall need to consider finite-dimensional truncations of the maps; clearly, these have the form of affine maps $\tilde{f}_{k}: \mathbb{R}^{n} \rightarrow \mathbb{R}^{n}$ where

$$
\tilde{f}_{k} x=T x+\beta_{k}
$$

where $T$ is an $n \times n$ diagonal matrix whose non-zero elements are given by $\left\{\lambda_{i}: i=\right.$ $1, \ldots n\}$, and for each input symbol $k, \beta_{k} \in \mathbb{R}^{n}$ has components $b_{i}^{(k)}$ with $i=1, \ldots, n$.

An upper bound. Proposition 3 immediately gives us an upper bound to the Hausdorff and box-counting dimensions of the attractor $K$ : recall that the number of maps $N_{S}$ in the IFS is just the number of symbols in the alphabet, and we have seen that the contractivity factor for each map is $\|S(\tau)\|=e^{-\tau\left(\rho+\pi^{2}\right)}$. (This is confirmed by (12) in the previous section: the largest diagonal element of $\bar{T}$ is $\lambda_{1}=e^{-\tau\left(\rho+\pi^{2}\right)}$.) Thus we have

$$
\operatorname{dim}_{H}(K) \leq \frac{\log N_{S}}{\tau\left(\rho+\pi^{2}\right)}
$$

Intuition suggests that this bound will be tightest for strongly contracting IFSs ${ }^{1}$. This situation corresponds to large values of the product $\tau\left(\rho+\pi^{2}\right)$-when for example, the symbol input rate is low or the leakage current dominates the current flow in the conductor. Conversely, in limits of potential technological interest-when the symbol rate is high and the cable is well-insulated - we suspect that the bound may be a considerable over-estimate.

This bound is based on quite non-specific information about the system. In the present case, since we are dealing with a system as nice as the cable equation, we can find the Hausdorff dimension of $K$ essentially exactly. It is instructive to compare the upper bound with the exact result.

The dimension of self-affine sets. We shall focus to begin with on a truncation of the infinite-dimensional IFS, $\left\{\tilde{f}_{k}: k=1, \ldots, N_{S}\right\}$, to some suitably large but finite number of dimensions $n$. The $\tilde{f}_{k}: \mathbb{R}^{n} \rightarrow \mathbb{R}^{n}$ are as defined in equation (13) and are affine maps. We shall refer to the attractor of this truncated IFS as $K_{n}$. A result originally due to Falconer [8] and extended by Solomyak [15] gives an explicit formula

\footnotetext{
${ }^{1}$ Roughly, the degree to which the covering balls intersect each other is a measure of the inefficiency of the cover, and generally these overlaps decrease as the contractivity of the maps increases
} 
for the Hausdorff dimension of the attractor of such a system, which works for 'most' systems (see below).

To describe this formula, we consider a linear map $T: \mathbb{R}^{n} \rightarrow \mathbb{R}^{n}$, and write the singular values of $T$, ordered to be a decreasing sequence, as $\sigma_{i}, i=1, \ldots, n$. The following function of $s(s \in[0, n])$ :

$$
\phi^{s}(T)=\sigma_{1} \sigma_{2} \ldots \sigma_{r-1} \sigma_{r}^{s-r+1}
$$

(where $1 \leq r \leq n$ is such that $r-1<s \leq r$ ), provides a multiplicative interpolation of the products of the singular values of $T$. It is strictly decreasing and continuous. For a general (self-affine) IFS, in which the affine maps may have different linear parts $\left\{T_{k}: k=1, \ldots, N_{S}\right\}$, the next step is to construct $\phi^{s}(T)$ for all products of the $T_{k}$ of length $q$ : Falconer introduces the $q$-th level sums

$$
\Sigma_{q}^{s}=\sum_{J_{q}} \phi^{s}\left(T_{i_{1}} \circ T_{i_{2}} \circ \ldots T_{i_{q}}\right)
$$

where $J_{q}$ denotes the set of all $q$-term sequences $\left(i_{1}, \ldots, i_{q}\right)$ with $1 \leq i_{j} \leq N_{S}$; he further defines $\Sigma_{\infty}^{s}$ to be $\lim _{q \rightarrow \infty}\left(\Sigma_{q}^{s}\right)^{1 / q}$ (this limit certainly exists because $\Sigma_{q}^{s}$ is a submultiplicative sequence).

We assume that $\Sigma_{\infty}^{n}<1$ (this can be ensured in the cable example by taking $n$ large enough, since we can make the product of singular values of $T_{k}$ arbitrarily small by doing this; see below). Since $\phi^{s}$ is a decreasing function of $s$, so is $\Sigma_{\infty}^{s}$, and so there exists a unique value of $s$, which we shall call $d\left(T_{1}, T_{2}, \ldots T_{N_{S}}\right)$, such that $\Sigma_{\infty}^{d}=1$.

Falconer proved the following theorem:

Theorem 4 (Falconer, Solomyak). Let $\left\{T_{k}: k=1, \ldots, N_{S}\right\}$ be linear contractions such that $\max _{1 \leq k \leq N_{S}}\left\|T_{k}\right\|<1 / 2$ and let $\left\{\beta_{k} \in \mathbb{R}^{n}: k=1, \ldots, N_{S}\right\}$ be vectors. If $K_{n}$ is the invariant set satisfying:

$$
K_{n}=\bigcup_{a=1}^{N_{S}}\left(T_{k}\left(K_{n}\right)+\beta_{a}\right)
$$

then $\operatorname{dim}_{H} K_{n}=\operatorname{dim}_{B} K_{n}=d\left(T_{1}, T_{2}, \ldots T_{N_{S}}\right)$ for almost all $\left(\beta_{1}, \beta_{2}, \ldots \beta_{N_{S}}\right) \in \mathbb{R}^{n N_{S}}$ in the sense of $n N_{S}$-dimensional Lebesgue measure.

Actually, Falconer's result was slightly weaker than this in that it assumed the condition on the norm of the linear maps was $\max _{1 \leq k \leq N_{S}}\left\|T_{k}\right\|<1 / 3$. Solomyak [15] showed that Falconer's dimension formula still works if $\max _{1 \leq k \leq N_{S}}\left\|T_{a}\right\|<1 / 2$, but that the result does not extend to $\max _{1 \leq k \leq N_{S}}\left\|T_{k}\right\|<1 / 2+\epsilon$ for any $\epsilon>0$.

The dimension of the cable equation attractor. Finding $d\left(T_{1}, T_{2}, \ldots T_{N_{S}}\right)$ (hereafter abbreviated to $d$ ) for the cable equation attractor is much simpler than for general self-affine IFS's, because the linear parts of the maps are all the same, and they are diagonal. Thus $T$ - see equation (13) - is diagonal, with the $k$ th diagonal element equal to $\lambda_{k}$, given in (12). We have, using (15),

$$
\Sigma_{q}^{s}=\sum_{J_{q}} \phi^{s}\left(T_{i_{1}} \circ T_{i_{2}} \circ \ldots T_{i_{q}}\right)=N_{S}^{q} \phi^{s}\left(T^{q}\right),
$$

and since $\phi^{s}\left(T^{q}\right)=\phi^{s}(T)^{q}$ (because $T$ is diagonal):

$$
\Sigma_{q}^{s}=N_{S}^{q} \phi^{s}(T)^{q} \quad \text { and } \quad \Sigma_{\infty}^{s}=N_{S} \phi^{s}(T)=N_{S} \lambda_{1} \lambda_{2} \ldots \lambda_{r-1} \lambda_{r}^{s-r+1} .
$$


In particular, $\Sigma_{\infty}^{n}=N_{S} \lambda_{1} \ldots \lambda_{n}$, confirming that $\Sigma_{\infty}^{n}<1$ for all sufficiently large $n$. The dimension $d$ is then the solution of $N_{S} \phi^{d}(T)=1$ and so

$$
d=r-1-\frac{\log \left(N_{S} \lambda_{1} \ldots \lambda_{r-1}\right)}{\log \lambda_{r}}=\frac{\tau\left(\pi^{2} / 6\right) r(r-1)(4 r+1)+\log N_{S}}{\tau\left(\rho+r^{2} \pi^{2}\right)}
$$

where we have used expression (12) for $\lambda_{k}$. This equation for $d$ is actually implicit, because we need $r$ to satisfy $r-1<d \leq r$; in practice we simply compute $d$ for successive values of $r$ until we find a value in the required range. Note that if $n$ is large enough for $\Sigma_{\infty}^{n}<1$ then the value of $d$ is independent of $n$.

Equation (16) gives the dimension of $K_{n}$ when the hypotheses of the FalconerSolomyak theorem are satisfied. The condition $\max _{1 \leq k \leq N_{S}}\left\|T_{k}\right\|<1 / 2$ required by the theorem reduces here to $\|T\|=\lambda_{1}<1 / 2$. If the condition is not satisfied, expression (16) provides an upper bound on the upper box-counting (and Hausdorff) dimension [8]; (the 'almost every' restriction on the $\beta_{i}$ 's is unnecessary for this). Physically, the norm condition can be thought of as a condition on the symbol input rate $\tau^{-1}<\left(\rho+\pi^{2}\right) / \log 2$. (Note that if there are only two symbols, $N_{S}=2$, then this condition implies that the right hand side of the inequality (14) is less than 1 ; hence we can put $r=1$ in (16) and find $d=\log 2 / \tau\left(\rho+\pi^{2}\right)$. Thus - for two symbols-(14) is actually an equality if the Falconer-Solomyak condition is satisfied.)

What can we say about the dimension of the attractor $K$ in the infinite-dimensional state space? Though we have seen that, for almost all choices of the translation vectors, the Hausdorff and box-counting dimensions of the projected attractor $K_{n}$ are equal to the constant $d$ for all sufficiently large $n$, we cannot immediately transfer this result to $K$, in part because it is unclear how to deal with the 'almost all' restriction in the infinite-dimensional case. However, we can still claim that $\operatorname{dim}_{H} K$ is unlikely to deviate significantly from $d$ :

Proposition 5. Given $\epsilon>0$ there is an $n \in \mathbb{N}$ such that, for (Lebesgue) almost all $b_{i}^{(k)}, 1 \leq i \leq n, 1 \leq k \leq N_{S}$ (and all $b_{i}^{(k)}, i>n, 1 \leq k \leq N_{S}$ ) the Hausdorff dimension of the attractor $\bar{K}$ of the IFS $\left\{\bar{f}_{1}, \ldots, \bar{f}_{N_{S}}\right\}$ on $\bar{l}_{2}$ satisfies

$$
d \leq \operatorname{dim}_{H} K<d+\epsilon
$$

where $d$ is given by (16).

Proof. The left inequality follows from the facts that $d=\operatorname{dim}_{H} K_{n}$ (for almost all $b_{i}^{(k)}$ ) for sufficiently large $n$, and that $\operatorname{dim}_{H} K_{n} \leq \operatorname{dim}_{H} K$ since $K_{n}$ is a projection of $K$.

The other inequality comes from noting that we can express $l_{2}$ as the product of $\mathbb{R}^{m}$ with the space $l_{2}^{(m)}$ of square summable sequences. (The superscript $(m)$ on $l_{2}^{(m)}$ indicates the role of this space as one part of the product forming $l_{2}$; as a set $l_{2}^{(m)}$ is of course identical to $l_{2}$. If we say $d_{E}$ is the Euclidean metric on $\mathbb{R}^{m}$ and $d_{m}$ is the $l_{2}$ metric on $l_{2}^{(m)}$, the state space $l_{2}$ has the product metric $\sqrt{d_{E}^{2}+d_{m}^{2}}$.) We can define, componentwise, IFS's on both $\mathbb{R}^{m}$ and $l_{2}^{(m)}$ using (11): the first is just the truncated IFS discussed above, and corresponds to $1 \leq n \leq m$ in (11); the second corresponds to $n>m$. Both of these IFS's have attractors: $K_{m}$ in the first case and $\hat{K}_{m}$ (say) in the second. It is clear that $K$ is contained in $K_{m} \times \hat{K}_{m}$, and so by a well-known formula for product sets [22] $\operatorname{dim}_{H} K \leq \operatorname{dim}_{H} K_{m}+\overline{\operatorname{dim}}_{B} \hat{K}_{m}$. From Proposition 3 we 


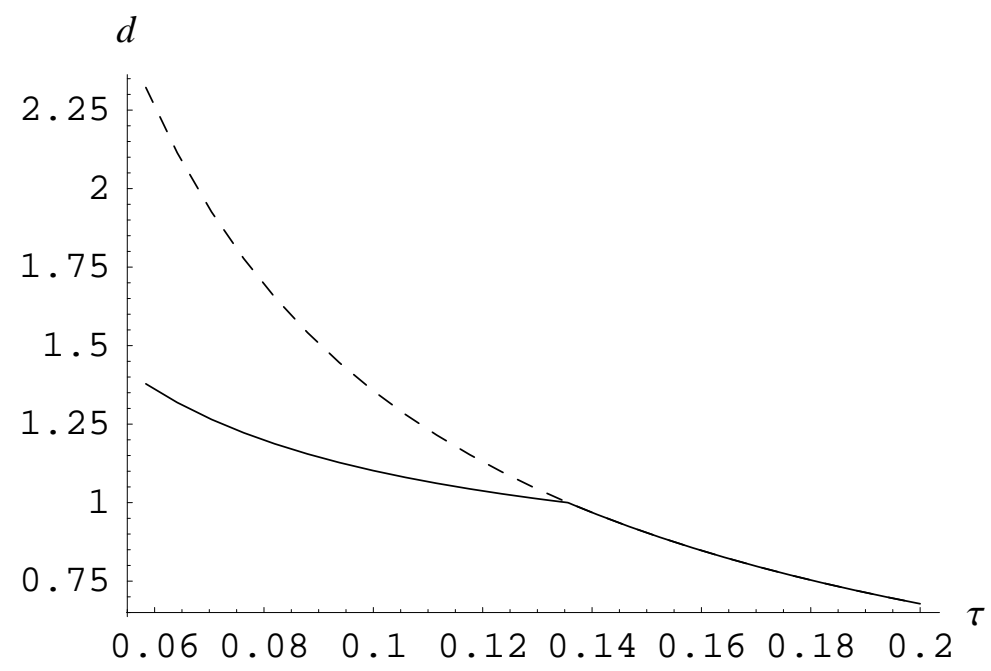

Figure 1. Solid curve: a plot of $\operatorname{dim}_{H} K$ versus $\tau$ with $\rho=2.0$ and $N_{S}=5$. Dashed curve: the upper bound from Proposition 3 .

have

$$
\overline{\operatorname{dim}}_{B} \hat{K}_{m} \leq \log N_{S} /|\log s|=\log N_{S} /\left|\log \lambda_{m+1}\right|=\log N_{S} /\left(\tau\left(\rho+m^{2} \pi^{2}\right)\right)
$$

and so by choosing $m$ large enough we can ensure $\overline{\operatorname{dim}}_{B} \hat{K}_{m}<\epsilon$. Again by choosing $m$ sufficiently large, and excluding a set of measure zero of components $b_{i}^{(k)}, 1 \leq i \leq m$, $1 \leq k \leq N_{S}$, we ensure $\operatorname{dim}_{H} K_{m}=d$, which proves the second inequality.

Noting that for bounded sets $A$ and $B$ we have $\overline{\operatorname{dim}}_{B}(A \times B) \leq \overline{\operatorname{dim}}_{B} A+\overline{\operatorname{dim}}_{B} B$, we see that the same argument shows that Proposition 5 remains true if we replace $\operatorname{dim}_{H}$ by $\overline{\operatorname{dim}}_{B}$.

Figure 1 shows the dependence of $d$ on the symbol period $\tau$; in this figure $N_{S}=5$ and $\rho=2.0$. ( $\tau$ is plotted from the minimum value of $\log 2 /\left(\rho+\pi^{2}\right) \approx 0.05$. $)$ Also shown is the upper bound given by Prop. 3; note that this is equal to $d$ for $d<1$.

\section{OBSERVABILITY OF THE CABLE EQUATION ATTRACTOR}

The usefulness of the IFS model of a digital channel rests on the 'observability' of the states of the system. At any given time we do not have full knowledge of the state: what we know instead is the value of some measurement function $h: M \rightarrow \mathbb{R}$. (This function is fixed, but may not be known in detail.) Thus if $x=x_{0}$ is the state at time zero, with $x_{1}, \ldots, x_{n-1}$ the subsequent states at times $\tau, 2 \tau, \ldots,(n-1) \tau$, we know the vector $\left(h\left(x_{0}\right), h\left(x_{1}\right), \ldots, h\left(x_{n-1}\right)\right)$. An observability result essentially asserts that (for $n$ sufficiently large) $x_{0}$ is uniquely determined by this delay vector (i.e. there is only one $x_{0}$ in $M$ that produces this particular delay vector). If such a result holds, then, the starting state $x$ may in principle be inferred from the (observed) delay vector. In practice this would require detailed knowledge of $h$ and of the system's dynamics, and is not something we are likely to attempt; but the uniqueness of $x$ may nonetheless be exploited - to make predictions for example - and this possibility has been explored extensively for deterministic dynamical systems [12].

For our digital channel (and IFS's generally), the sequence $x_{1}, x_{2}, \ldots$, depends not only on $x$ but also the input sequence of symbols. In particular, suppose the first 
$n-1$ symbols form the $(n-1)$-tuple $\left(i_{1}, \ldots, i_{n-1}\right)$ with $1 \leq i_{j} \leq N_{S}$ (and the maps in the IFS are, as usual, $f_{k}, k=1, \ldots N_{S}$ ), then the resulting delay vector will be

$$
\Phi_{\omega}(x)=\left(h(x), h\left(f_{i_{1}}(x)\right), h\left(f_{i_{2}} \circ f_{i_{1}}(x)\right), \ldots, h\left(f_{i_{n-1}} \circ \ldots \circ f_{i_{1}}(x)\right)\right)
$$

(where we have denoted $\left(i_{1}, \ldots, i_{n-1}\right)$ by $\omega$ ). The delay map $\Phi_{\omega}$ maps the state space $M$ to $\mathbb{R}^{n}$. The condition of observability can be expressed by saying that $\Phi_{\omega}$ is injective, either on the whole of $M$ or more likely on some suitable subset - the subset of interest to us is the attractor of the system.

Of course it is easy to think of measurement functions (e.g. constant functions) for which $\Phi_{\omega}$ cannot possibly be injective, so the best we can hope for is that some large collection of measurement functions produce injections. Results along such lines, for nonlinear autonomous systems (corresponding to $N_{S}=1$ ) first appeared in the 1980's [1, 18] (for IFSs see [16]), but of more relevance to the current problem is the work of Sauer, Yorke and Casdagli [17]. These authors showed that, given a compact set $A \subset \mathbb{R}^{k}$, invariant under a diffeomorphism $f$ and having box-counting dimension $d$ then, under some conditions on the periodic orbits of $f$, almost all smooth mappings $h: \mathbb{R}^{k} \rightarrow \mathbb{R}$ produce delay maps that are injective on $A$, so long as the number of delays $n$ is greater than $2 d$. The sense given to 'almost all' here is that of 'prevalence' - a generalization of Lebesgue 'almost all' to infinite-dimensional spaces. In practice the authors proceed by choosing a fixed set of functions $h_{i}: \mathbb{R}^{k} \rightarrow \mathbb{R}, i=1, \ldots, m$ (in fact they use a basis for the polynomials of degree up to $2 n$ in $k$ variables) and show that, for any smooth function $h_{0}$, the measurement function

$$
h_{a}(x)=h_{0}(x)+\sum_{i=1}^{m} a_{i} h_{i}(x)
$$

(where $a=\left(a_{1}, \ldots, a_{m}\right) \in \mathbb{R}^{m}$ ) produces an injection for almost all $a$ (in the Lebesgue sense on $\mathbb{R}^{m}$ ). Robinson [14] generalized this to allow $A$ to be a compact subset of an infinite-dimensional space (but still with finite box-counting dimension) and $f$ to be an injective Lipschitz map. (In fact, much more is proved in [17] than we have saidand not all of it generalizes to infinite dimensions - but it is the injectivity property that we are concerned with here.) The number of delays required is now somewhat higher: for Hilbert spaces it is $n>(2+\hat{t}) d$ where $\hat{t}$ is the 'thickness exponent' of $A$, see [14] for a definition. (A similar result holds for general Banach spaces, but the number of delays needed is higher again.)

Robinson's result is quite close to showing that, for a fixed $\omega, \Phi_{\omega}$ is injective on the compact invariant set $A$; the difference between $\Phi_{\omega}$ and the delay map considered in [14] is that the former involves several maps $f_{k}$, not just one (all are taken to be injective and Lipschitz). Nevertheless the argument can be carried over largely without change. In particular it shows that there is a bounded linear function $L: X \rightarrow$ $\mathbb{R}^{N}$ from the infinite-dimensional state space $X$ to a finite-dimensional space $\mathbb{R}^{N}$ that is injective on $A$, and further that there are $\alpha$-Hölder maps on $A^{\prime}=L A, g_{k}=$ $L \circ f_{k} \circ L^{-1}$ constituting an isomorphic IFS on $A^{\prime}$.

The only addition necessary to Robinson's proof concerns the conditions on the periodic orbits of $f$. These conditions arise from the fact that there can be pairs $(x, y)$ of states in $A$ for which the trajectory segments $x=x_{0}, x_{1}, \ldots, x_{n-1}$ and $y=$ $y_{0}, y_{1}, \ldots, y_{n-1}$ have points in common (either $x_{i}=x_{j}$, or $x_{i}=y_{j}$, for some $i \neq j$; note that the injectivity of the maps means that $x \neq y$ prohibits $x_{i}=y_{i}$ ). For such pairs certain elements in the corresponding delay vectors must be equal whatever the 
measurement function, and for the arguments in [17] to work, these pairs must not occur too frequently - there are restrictions on their box-counting dimension (as a subset of $A \times A$ ). For the autonomous case the only points that cause problems lie on periodic orbits of low period, but in the IFS case the possibilities for equalities in the trajectory segments are much more extensive. We can specify the needed restrictions for the IFS case as follows: for each pair $(x, y)$ we say the 'length' of $(x, y)$ is the number of distinct elements in the union of the trajectory segments $\left\{x_{0}, \ldots, x_{n-1}\right\}$ and $\left\{y_{0}, \ldots, y_{n-1}\right\}$ (thus for $x \neq y, 2 \leq$ length $\leq 2 n$ ). Define $A_{l} \subset A \times A$ by

$$
A_{l}=\{(x, y) \mid x \neq y ; \text { length of }(x, y)=l\} \text {, }
$$

then the required conditions are that $\operatorname{boxdim}\left(A_{l}\right)<l /(2+\hat{t})$ for $l=2, \ldots, 2 n$.

Let us verify that these conditions are satisfied by the cable equation system; this follows readily from the fact that in this system the maps have the affine form $f_{k}(x)=T x+b^{(k)}$, with common linear part $T$, see (11) and (12) (we have dropped the overbars for convenience). The $l=2 n$ case is automatically satisfied by the choice $n>(2+\hat{t}) d$, so we need only consider $l<2 n$. For the length of a pair $(x, y)$ to be less than $2 n$ there must be an equality of the form $x_{i}=x_{j}$ (or $y_{i}=y_{j}$ ) or $x_{i}=y_{j}$. Suppose first that $x_{i}=x_{j}$ (and take $j>i$ ); then $T^{i} x+b_{1}=T^{j} x+b_{2}$ for some (constant) vectors $b_{1}$ and $b_{2}$, and so $T^{i}\left(I-T^{j-i}\right) x=b_{2}-b_{1}$. This can only happen if there is some $\hat{b}$ such that $T^{i} \hat{b}=b_{2}-b_{1}$; the injectivity of $T$ means that $\hat{b}$ is unique, and further that $\left(I-T^{j-i}\right) x=\hat{b} \cdot I-T^{j-i}$ is also injective, so there is (at most) a single solution for $x$. (Call this solution $x_{*}$.)

If, in addition to $x_{i}=x_{j}$, an equation of the form $y_{i^{\prime}}=y_{j^{\prime}}$ is also satisfied, then there is at most a single possibility for the pair $(x, y)$; such cases can make no contribution to boxdim $\left(A_{l}\right)$ for any $l$, so we move on to the case where $y_{0}, \ldots, y_{n-1}$ are all distinct. The length of $(x, y)$ is then at least $n$. All the pairs satisfying $x_{i}=x_{j}$ lie in the set $\left\{x_{*}\right\} \times A$, the box-counting dimension of which is clearly $d$, and hence less than $n /(2+\hat{t})$, as required.

Note that any collection of equalities between elements of the two trajectory segments that includes an equation of the form $x_{i}=x_{j}$ falls into one of the two cases considered above: either there is an equation $y_{i^{\prime}}=y_{j^{\prime}}$ (and so at most one possible pair $(x, y)$ satisfying the collection), or all the $y_{i^{\prime}}$ are distinct. So the above argument deals with all such collections.

Finally we consider the case $x_{i}=y_{j}$. Since cases where equations such as $y_{i}=y_{j}$ hold have already been dealt with we may assume $y_{0}, \ldots, y_{n-1}$ are all distinct and hence that the length is at least $n$. For the cable system $x_{i}=y_{j}$ becomes $T^{i} x+b_{1}=$ $T^{j} y+b_{2}$, and using the injectivity of $T$ as before we conclude that $x=T^{j-i} y+\hat{b}$ (for some constant $\hat{b})$. Hence $(x, y)$ lies on the graph of a Lipschitz function $y \mapsto T^{j-i} y+\hat{b}$ over $A$; the box-counting dimension of this graph can be no larger than that of $A$ (i.e. $d)$, which is less than $n /(2+\hat{t})$, as required.

Robinson's proof therefore shows that, for a fixed $\omega$, the delay map $\Phi_{\omega}$ is injective on the cable system attractor $A$, for almost all measurement functions, so long as we use enough delays. (We have said nothing about the thickness exponent, but in any case (see [9]) we have $\hat{t} \leq \overline{\operatorname{dim}}_{B} A$.) This is the observability result we were seeking: it means that the IFS model of the cable equation system has some of the features of the IFS models described in [5], and we can attempt to use it in a similar way, to try to identify the transmitted symbol sequence. 


\section{CONCLUSIONS AND IMPLICATIONS}

Thinking about how a digital signal is transmitted through a channel leads quite directly to a mathematical model in the form of an iterated function system. Such a model suggests that the output from the channel can be used to reconstruct aspects of the channel's behaviour, and the forcing to which it is subjected - that is, the transmitted symbol sequence. This suggestion was put on a firm foundation in [5] for the case of finite-dimensional systems. Approaches to performing the reconstruction and identifying the transmitted signal are also discussed there. We have now seen that much of what is said in [5] applies to an infinite-dimensional system; we have focused on the cable equation, but it seems likely that the same general ideas will be applicable to other systems, including certain kinds of nonlinear system, and should provide the basis for an inclusive theory of digital channels.

Development of practical algorithms based on the IFS model awaits further work, and will need to address a number of matters to do with reconstruction using the measured output. For example: although we have seen that the image $\Phi_{\omega} A$ contains no self-intersections, we have not shown that images of $A$ under delay maps corresponding to different $\omega$ 's have no intersections - and indeed such intersections can exist. These intersections can even be persistent under perturbations of the maps $f_{k}$; but this situation exists in the finite-dimensional case as well - see [5] for a discussion.

Further, the delay maps applied to the infinite-dimensional system retain the injectivity properties of the finite-dimensional ones, but not the smoothness propertiesalthough to what extent these are lost in any given case is unclear, as are the implications of the loss for the ease of recovering the transmitted signal. (Some experiments may illuminate these issues.)

Most current interest in the cable equation itself centres around the modelling of neural projections (dendrites and unmyelinated axons). While our discussion is not really intended to provide insights into such modelling, we note that the viewpoint taken here allows the spatially distributed nature of neural input to be linked to dynamical behaviour in a very direct way (and perhaps even the digital signalling idea captures something of the nature of input by regular pulse trains), so a more realistic model built along such lines seems possible, and the idea of an attractor may have some relevance. Of course, the cable equation is only relevant to subthreshold membranes, but it is interesting to note that even while remaining subthreshold the state of the membrane is in principle capable of storing information about the history of inputs to the cell.

Although we have emphasized the digital signal channel as motivation for the models we have described there may be scope for applying them in other situations: what we would look for are systems displaying internal (probably nonlinear) dynamics and subject to external forcing that might be reasonably modelled as an IFS; with sufficient ingenuity the range of possible applications could be rather wide.

\section{ACKNOWLEDGEMENT}

This research was partially support by Engineering and Physical Sciences Research Council grant EP/E050441/1 (CICADA: Centre for Interdisciplinary Computational and Dynamical Analysis) 


\section{REFERENCES}

[1] D. Aeyels (1981) Generic Observability of Differentiable Systems, SIAM J. Control Optim., 19, 595-603

[2] M. Barnsley (1988). Fractals Everywhere, Academic Press Inc., San Diego.

[3] M.F Barnsley, A.N. Harrington (1985) A Mandelbrot Set for Pairs of Linear Maps, Physica D, 15D, 421-432

[4] A. Belleni-Morante (1979). Applied Semigroups and Evolution Equations, Oxford University Press, Oxford UK.

[5] D.S. Broomhead, J.P. Huke, M.R. Muldoon, J. Stark (2004) Iterated Function System Models of Digital Channels Proc. Roy. Soc. Lond. A 460, 3123-3142

[6] P. Dayan and L.F Abbott (2001). Theoretical Neuroscience, The MIT Press, Cambridge USA.

[7] K. Falconer (1990) Fractal Geometry: Mathematical foundations and applications, New York: John Wiley and Sons.

[8] K.J. Falconer (1988). The Hausdorff dimension of self-affine fractals, Math. Proc. Camb. Phil. Soc., 103, 339-350.

[9] B.R. Hunt, V.Y. Kaloshin (1999) Regularity of embeddings of infinite-dimensional fractal sets into finite-dimensional spaces, Nonlinearity, 12, 1263-1275.

[10] J.E. Hutchinson, (1981) Fractals and self-similarity, Indiana Math. J., 30, 713-747.

[11] W.C. Johnson (1963). Transmission Lines and Networks, McGraw-Hill, Singapore.

[12] H. Kantz, T. Schreiber (1997). Nonlinear time series analysis, Cambridge University Press, Cambridge.

[13] A. Pazy (1983). Semigroups of Linear Operators and Applications to Partial Differential Equations, Springer, New York.

[14] J.C. Robinson (2005) A topological delay embedding theorem for infinite-dimensional dynamical systems, Nonlinearity, 18, 2135-2143

[15] B. Solomyak, (1998), Measure and dimension for some fractal families, Math. Proc. Camb. Phil. Soc. 124, 531-546

[16] J. Stark, D.S. Broomhead, M.E. Davies and J.P. Huke (2003) Delay Embedding for Forced Systems: II, Stochastic Forcing, J. Nonlinear Sci., 13, 519-577

[17] T. Sauer, J.A. Yorke and M. Casdagli (1991) Embedology, J. Stat. Phys., 65, 579-616.

[18] F. Takens, (1981) 'Detecting strange attractors in turbulence', in Dynamical Systems and Turbulence, (Springer Lecture Notes in Mathematics, vol. 898) edited by D.A. Rand and L.-S. Young, Springer-Verlag, Berlin.

[19] W. Thomson (1855) On the Theory of the Electric Telegraph, Proc. Roy. Soc. 7, 382-399

[20] H.C. Tuckwell (1988). Introduction to Theoretical Neurobiology, vol. 1: Linear Cable Theory and Dendritic Structure, Cambridge University Press, Cambridge UK.

[21] J.A. Walker (1980). Dynamical Systems and Evolution Equations, Plenum Press, New York.

[22] H. Wegmann (1971) Die Hausdorff-Dimension von kartesischen Produkten metrischer Räume, J. reine angew. Mathematik 1971, 46-75

The School of Mathematics, University of Manchester, Oxford Road, Manchester M13 9PL, UK 